\title{
Sieben auf einen Streich - Agave blüht mit mehrstängeligem Blütenstand in einem Privatgarten
}

\author{
Hilke Steinecke
}

\begin{abstract}
The century plant (Agave americana) requires particular wintertime protection when grown under Central European climatic conditions. A unique 50-year-old specimen cultivated at Bruchköbel near Hanau, Hesse, set flower in the summer of 2015. Apparently, an early injury inflicted to the plant had divided the inflorescence axis. Seven closely growing shafts developed with some 5000 individual flowers.
\end{abstract}

\section{Zusammenfassung}

Amerikanische Agaven (Agave americana) sind bei uns nicht winterhart. Mit entsprechendem Schutz können sie auch draußen ausgepflanzt überleben. Eine rund 50 Jahre alte, im Garten ausgepflanzte und mit Winterschutz versehene Agave americana blühte im Sommer 2015 in einem Privatgarten in Bruchköbel bei Hanau. Aufgrund einer vermutlich früh aufgetretenen Verletzung hatte sich die Haupt-Blütenstandsachse geteilt. Dicht gedrängt erschienen sieben Stängel mit insgesamt rund 5000 Blüten.

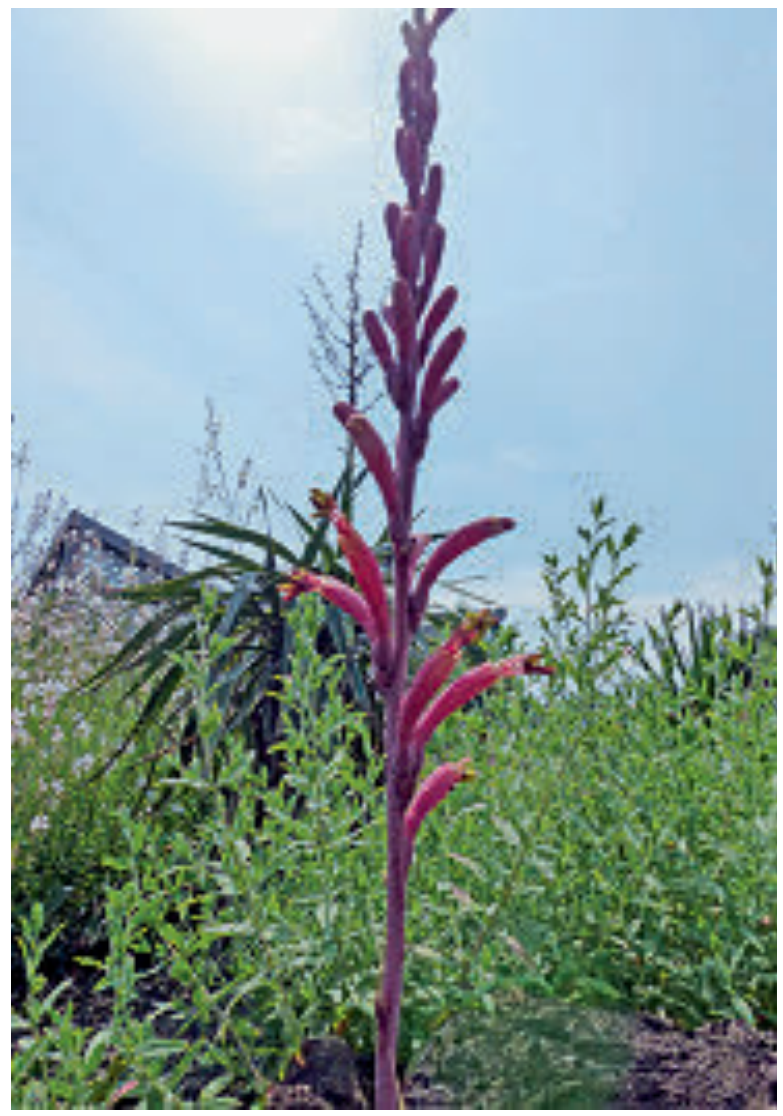

\section{Agaven, klein und groß}

Agaven sind Rosettenpflanzen, von denen einige nur wenige Zentimeter breit und hoch werden, andere dagegen mehrere Meter breite Rosetten bilden und über $10 \mathrm{~m}$ hohe Blütenstände entwickeln können. Von den rund 200 Arten sind nur wenige winterhart. Beispiele hierfür sind im Amerikateil der Steppenanlage im Palmengarten zu sehen. Diese kleinen Agaven ertragen ausgepflanzt im Freien mäßige Fröste, werden im Winter jedoch durch Plexiglasplatten vor Regen und daraus resultierender Fäulnis geschützt. Die meisten Agaven sind jedoch frostempfindlich. Sie mögen es gern, wenn sie im Sommer im Freien stehen, müssen jedoch zum Winter reingeholt werden.

Relativ bald nach der Entdeckung Amerikas gelangten Agaven als Zierpflanzen nach Europa. Im 17. und 18. Jahrhundert waren Agaven beliebte Modepflanzen, die in keinem fürstlichen Garten fehlen durften. Im Kübel gehaltene, blühende Agaven galten nördlich der Alpen als etwas ganz Außergewöhnliches und Wertvolles. Die Entwicklung des Blütenstandes und die Zahl der Blüten wurden genauestens protokolliert. Pro Agave können durchaus 10000 Blüten gebildet werden. Die blühenden Pflanzen wurden gezeichnet, zudem wurden passend zum Blühereignis Medaillen geprägt oder Lobgedichte verfasst. Diese sollten dem stolzen Besitzer der blühenden Agave viel Glück bringen.

\section{Vom Fürstengarten auf die Fensterbank} Mittlerweile sind Amerikanische Agaven (Agave americana) mit ihren bis $12 \mathrm{~m}$ hohen Blütenständen im Mittelmeerraum oder auch auf den Kanaren ein alltägliches Bild. Auch als Zimmerpflanzen sind sie bei uns sehr beliebt, man 


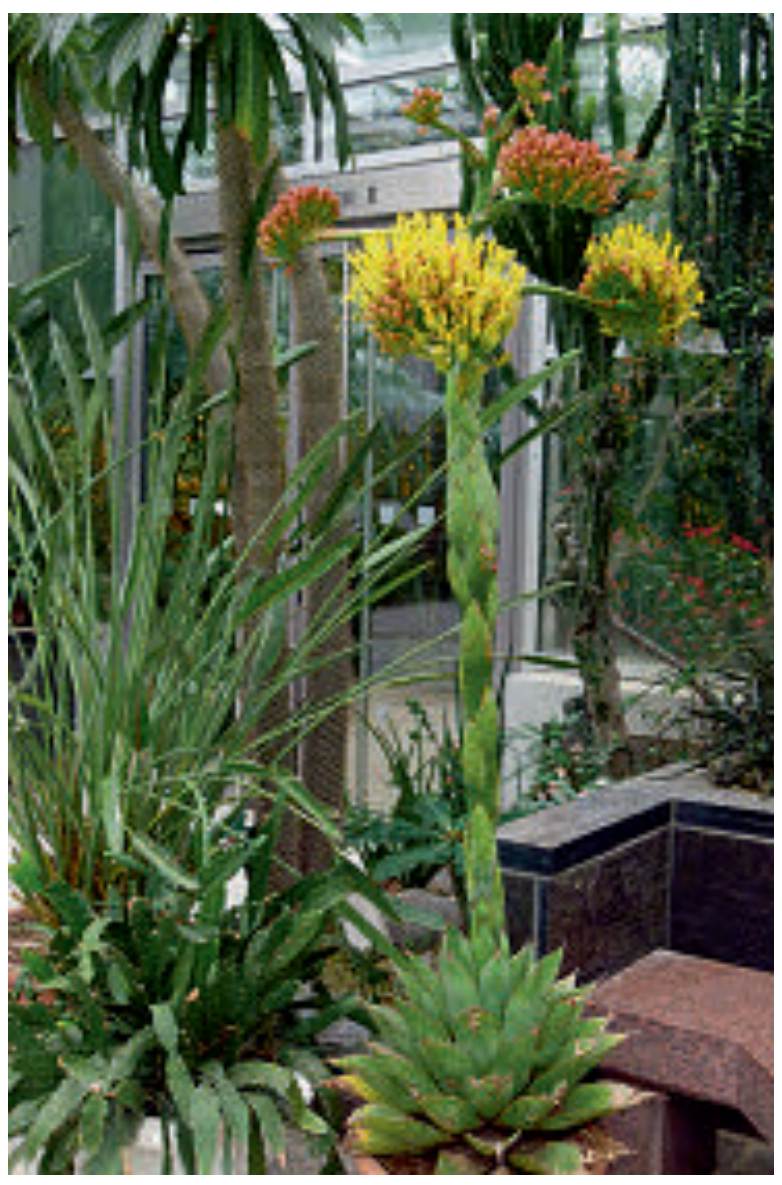

muss sie schließlich kaum gießen und sie gehen nicht so schnell ein. Wenn die Agaven nicht gerade im Sommerhalbjahr im Freien stehen, fristen sie häufig ihr Dasein in einem relativ kleinen Topf auf der Fensterbank, bis sie für die Platzverhältnisse in der Wohnung oder im Wintergarten zu groß geworden sind. Häufig werden sie entsorgt, bevor sie überhaupt zur Blühreife gekommen sind.

\section{Nach einem halben Jahrhundert endlich blühend}

Auch Elsa und Willi Plein aus Bruchköbel bei Hanau hielten seit etwa 50 Jahren eine Agave americana im Topf. Im Sommer kam sie in den Garten, den Winter verbrachte sie vor der

Abb. 1 (Seite 100): Agave polianthiflora ist eine kleine Art, die bei uns winterhart ist.

Abb. 2 (links): Agave parrasana im Tropicarium.

Abb. 3 (rechts): Die zu groß gewordene Agave wird im Mai 2001 in den Garten gebracht.

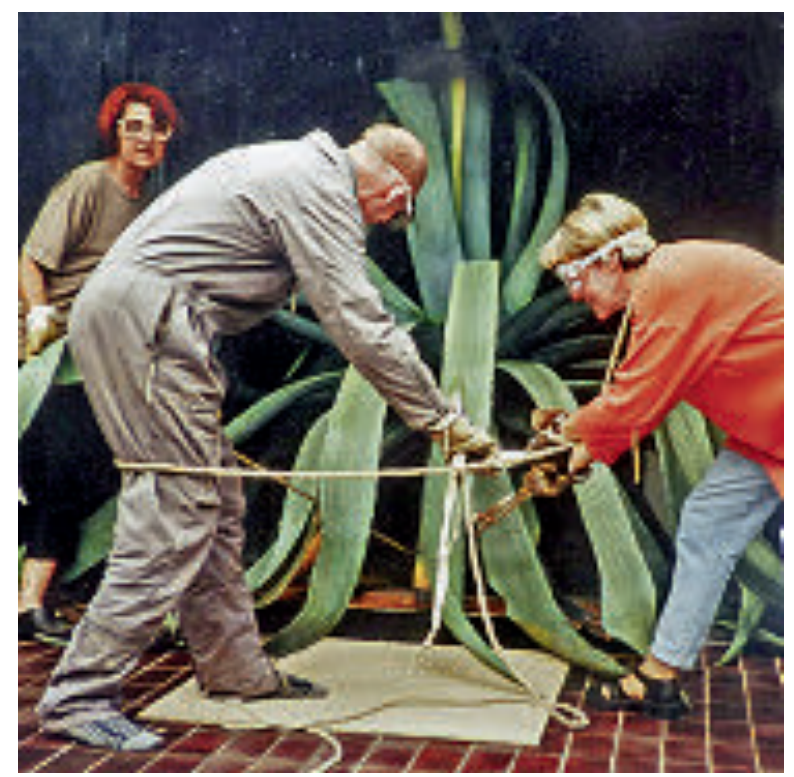

Terrassentür im Wohnzimmer. Mit den Jahren war der jährliche Transport der Agave nach draußen schwer geworden und nicht mehr möglich. Willi Plein wollte die zu groß gewordene Pflanze aber nicht abgeben, sondern hoffte, sie eines Tages in Blüte zu erleben.

Im Jahre 2001 wog die Agave mitsamt Topf, Erde und Wurzeln etwa $500 \mathrm{~kg}$. Es wurde hinten im Garten ein 3,20 m hoher Verschlag mit einer Grundfläche von 3,20 m mal 3,20 m gebaut, ähnlich wie ein Foliengewächshaus. In ihr neues Domizil wurde die Agave mit einem kleinen Bagger transportiert. Im Winter wurde das Agavenhaus mit Treibhausfolie verschlossen und die Seitenplatten zusätzlich mit Styropor isoliert. Ein Heizlüfter mit Ventilator, der bei einer Temperatur von $2^{\circ} \mathrm{C}$ eingeschaltet wurde, sorgte für Frostfreiheit. Mit diesem Schutz überlebte die Agave sogar die Extremwinter 2010-2012. Beinahe hätte die Agave allerdings in einer eisig frostigen Winternacht 2003 Schaden genommen, als Pleins erst spät nachts nach Hause kamen und der Stecker der Heizung versehentlich aus der Steckdose gezogen war. Die Außentemperatur betrug $-10{ }^{\circ} \mathrm{C}$, im Gewächshaus war die Temperatur bereits auf $-2{ }^{\circ} \mathrm{C}$ abgesunken. Aus Besorgnis, dass die Heizung das Gewächshaus nicht schnell genug aufheizen und die Agave erfrieren könne, wurden ganz schnell Eimer mit heißem Wasser in das Agavenhaus geschleppt, um den Aufheizvor- 

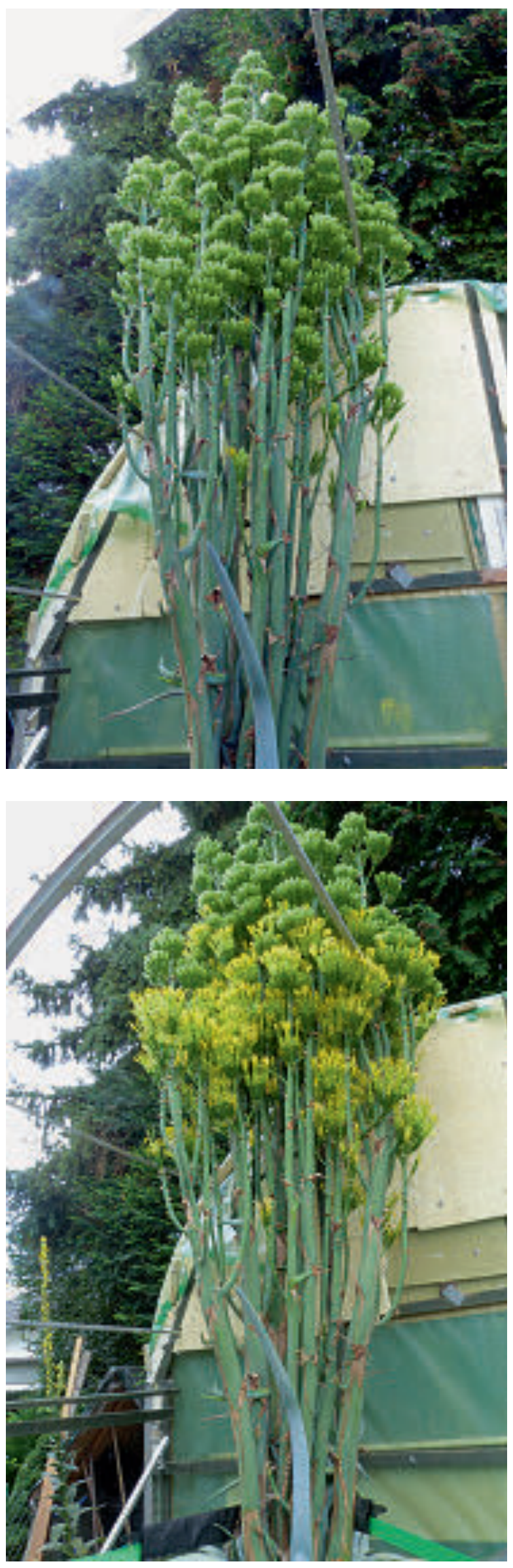

gang zu beschleunigen. Äußerlich hatte die Agave davon keinen Schaden genommen, allerdings wuchs sie im folgenden Jahr zunehmend schiefer und kippte nach zwei Jahren um, sodass sie $2005 \mathrm{im}$ „Agavenhaus“ nun richtig in den Boden ausgepflanzt und dabei auch wieder etwas aufgerichtet wurde. Die Agave war so schwer, dass sie mit einem Seilzug angehoben werden musste, um in das Pflanzloch gesetzt werden zu können. Die Blätter entwickelten sich gut und wuchsen zunächst durch das Foliendach, später passten sie sich in ihrer Krümmung an die Dachschräge an.

\section{Sieben auf einen Streich}

Bei der Agave der Familie Plein zeigte sich ein ganz besonderes Phänomen, das nur selten auftritt. Vermutlich hatte die Anlage des Blütenstandes im ganz frühen Entwicklungsstadium Schaden genommen, sodass es durch unkontrolliertes Wachstum des Gewebes bei der „Wundheilung“ zu einer Teilung der Blütenstandsachse bzw. dessen Vegetationskegels kam. Ab Mitte Mai 2015 entwickelte sich ein mehrfach geteilter Blütenstand mit sieben kräftigen Stängeln aus der Mitte der rund $3 \mathrm{~m}$ breiten Blattrosette.

Auch wenn es sich um eine Missbildung handelte, war der Blütenstand sehr beeindruckend. Sie wirkten sehr bizarr, ähnlich wie riesige

Abb. 4 (oben): Der Blütenstand mit zahlreichen Knospen.

Abb. 5 (unten): Die Blüten des siebenachsigen Blütenstandes öffnen sich von unten nach oben.

Abb. 6 (Seite 103 oben links): Blick von oben durch die Blüten auf die Blattrosette.

Abb. 7 (Seite 103 oben rechts): Nur mit der Leiter ist der obere Teil des Blütenstandes zu erreichen.

Abb. 8 (Seite 103 Mitte links): Der dichte Blütenstand mit massenhaft gelben Blüten.

Abb. 9 (Seite 103 Mitte rechts): Die Staubblätter ragen aus der Blütenkrone heraus.

Abb. 10 (Seite 103 unten links): Einige Blüten haben Früchte angesetzt.

Abb. 11 (Seite 103 unten links): Eine Gruppe aufgeblühter Blüten mit exponierten Antheren. 

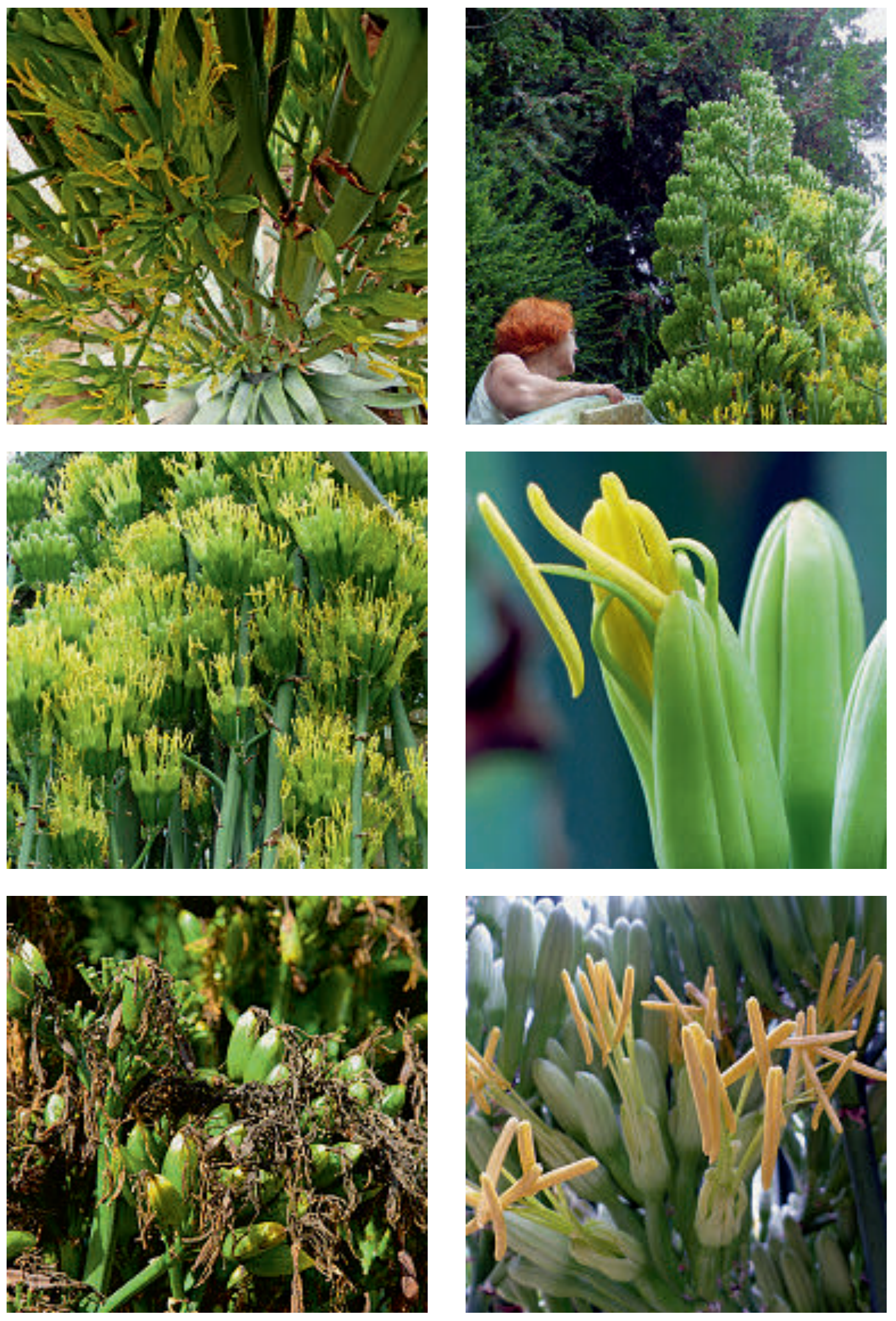


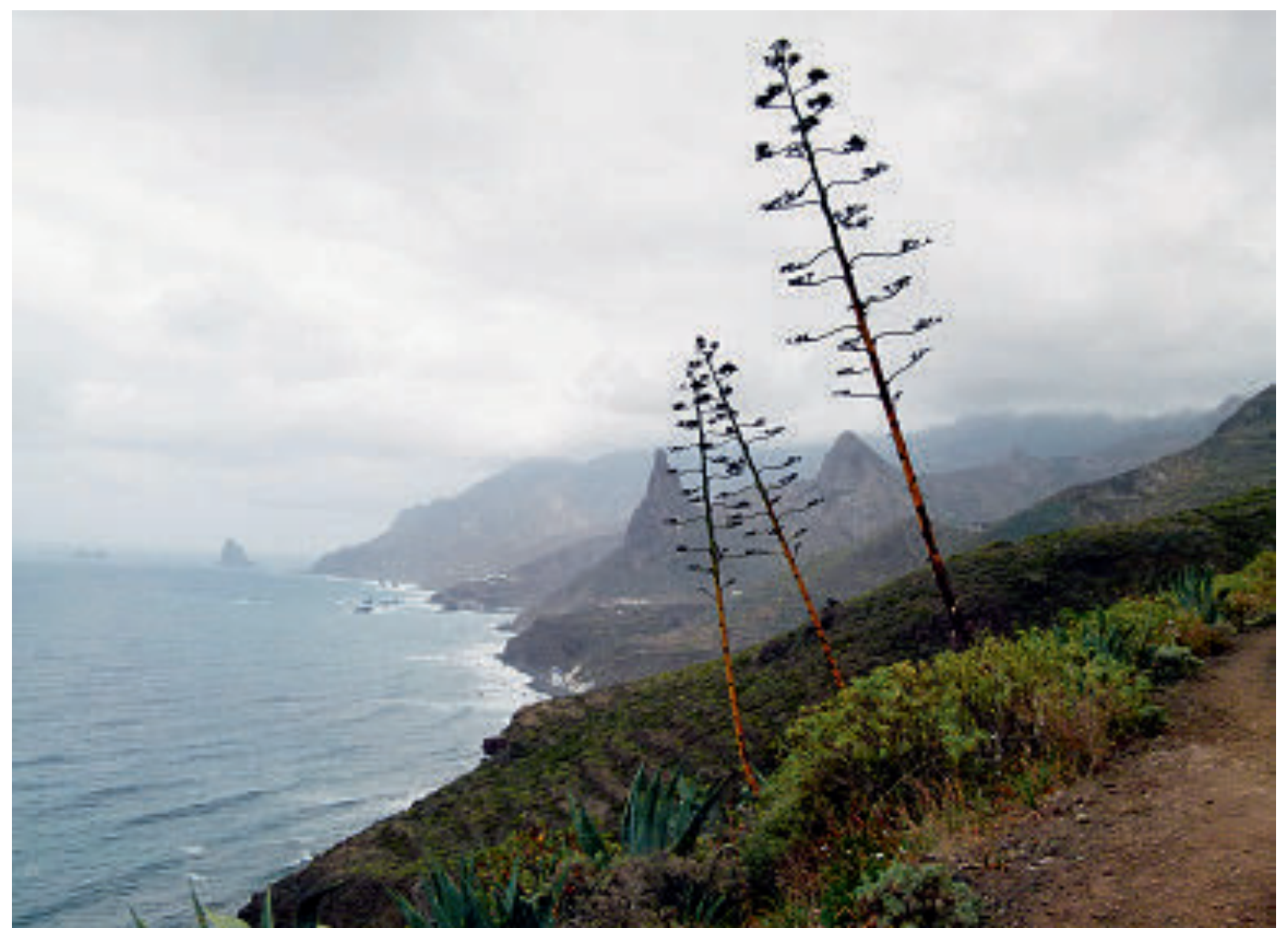

Blumen in einer etwas zu engen Vase. Sie erreichten schließlich eine Höhe von rund $5 \mathrm{~m}$. Dass es sich um eine irreguläre Entwicklung der Blütenstände handelte, zeigte sich daran, dass im unteren Bereich der Blütenstandsachsen die normalerweise hier nur schuppenartig ausgeprägten Hochblätter an Miniaturausgaben der sukkulenten und am Rand gezähnten Blätter erinnerten.

In den heißen Sommertagen von Mitte Juli bis Mitte August 2015 blühten innerhalb weniger Wochen alle der geschätzt etwa 5000 Blüten der Agave sukzessiv von unten nach oben auf.

Agaven werden in ihrer Heimat je nach Art von Insekten, Fledermäusen oder Vögeln bestäubt. Hier waren es unzählige Bienen und Wespen, die scharenweise die Blüten aufsuchten, um den reichlich vorhandenen Nektar zu trinken. Im Herbst schließlich war die Blühphase endgültig vorbei, zum Winter wurden die alten Stängel abgeschnitten. Die Mutterrosette der Agave stirbt bekanntlich nach dem Blühen und Fruchten ab. Pleins kultivierten aber einen Ableger ihrer alten Agave weiter. Ein Kindel hat bereits vor einiger Zeit einen Platz im Haus des Sohnes gefunden und die Enkel sind ebenfalls zu eifrigen „Agavengärtnern“ geworden.

Dieses Beispiel soll dazu anregen, bei ausreichend Platz und Möglichkeit eines Winterschutzes auch einmal auszuprobieren, eigentlich nicht winterharte Amerikanische Agaven auszupflanzen. Wenn man dann noch viel Geduld mitbringt und etwas Glück hat, kann vielleicht auch zu Hause einmal eine blühende Agave begeistern. Besonders in heißen, sonnigen Sommern wird bei entsprechend alten Agaven die Entwicklung von Blüten gefördert. Im Palmengarten sind regelmäßig, sowohl in den Gewächshäusern angepflanzte Exemplare als auch im Kübel gehaltene Individuen verschiedener Agaven-Arten, mit Blüten zu sehen.

Abb. 12 (oben): Alte, normal ausgebildete Fruchtstände von auf Teneriffa verwilderten Amerikanischen Agaven. 